AperTO - Archivio Istituzionale Open Access dell'Università di Torino

\title{
Antileishmanial activity of HIV protease inhibitors
}

\section{This is the author's manuscript}

Original Citation:

Availability:

This version is available http://hdl.handle.net/2318/42372

since

Terms of use:

Open Access

Anyone can freely access the full text of works made available as "Open Access". Works made available under a Creative Commons license can be used according to the terms and conditions of said license. Use of all other works requires consent of the right holder (author or publisher) if not exempted from copyright protection by the applicable law. 


\title{
Antileishmanial activity of HIV protease inhibitors
}

\author{
Dianella Savoia $^{\mathrm{a}, *}$, Tiziano Allice $^{\mathrm{a}}$, Pier-Angelo Tovo ${ }^{\mathrm{b}}$ \\ ${ }^{a}$ Laboratory of Microbiology, Department of Clinical and Biological Sciences, \\ University of Torino at S. Luigi Gonzaga Hospital, 10043 Orbassano, Torino, Italy \\ b Department of Paediatric Sciences, University of Torino, Italy \\ Received 10 January 2005; accepted 6 April 2005
}

\begin{abstract}
The proteasomes of some protozoa are possible targets for chemotherapy. Leishmaniasis is a major health problem in human immunodeficiency virus (HIV) co-infected subjects. Two HIV protease inhibitors (PI), indinavir and saquinavir, have been shown to block proteasome functions; we therefore investigated their effects on the growth of two Leishmania spp. (Leishmania major and Leishmania infantum). After $24 \mathrm{~h}$ of treatment, both drugs exhibited a dose-dependent antileishmanial activity, with $50 \%$ lethal dose $\left(\mathrm{LD}_{50}\right)$ values of, respectively, $8.3 \mu \mathrm{M}$ and $7 \mu \mathrm{M}$ on $L$. major; minor activity was observed on $L$. infantum. These results add new in vitro insights into the wide-spectrum efficacy of PI and suggest studying their action on amastigote forms of leishmania within macrophages in order to validate their potential contribution against opportunistic infections in treated seropositive patients.
\end{abstract}

(C) 2005 Elsevier B.V. and the International Society of Chemotherapy. All rights reserved.

Keywords: Leishmania major; Leishmania infantum; Protease inhibitors; Proteasomes

\section{Introduction}

Leishmaniasis currently affects an estimated 12 million people, in every continent except Australia and Antarctica. Moreover, the emergence of visceral leishmaniasis as an opportunistic infection [1], particularly in patients infected with human immunodeficiency virus (HIV), has given rise to the need for new therapeutic strategies. The control of leishmania infection is T-cell dependent and requires a Th1 response. There has therefore been an increased incidence of kala azar in HIV co-infected patients in Bihar (India) [2], and in the Mediterranean basin up to $9 \%$ of acquired immune deficiency syndrome (AIDS) patients suffer from newly acquired or reactivated visceral leishmaniasis [3]. Treatment with antimonials, the mainstay of therapy, is less satisfactory in immunocompromised patients than in immunocompetent subjects [1]. New therapies, such as lipid formulations of amphotericin B or miltefosine, are very expensive. Therefore, there is a pressing need for new antileishmanial drugs. The incidence of HIV-Leishmania co-infections has been

\footnotetext{
* Corresponding author. Tel.: +39011670 5427; fax: +390119038639.

E-mail address: dianella.savoia@unito.it (D. Savoia).
}

decreasing since the introduction of highly active antiretroviral therapy (HAART) [4,5]. Some antiretroviral agents exhibit significant inhibitory effects on purified human proteasome functions [6,7]. In recent years several studies have documented the antiprotozoal activity of some proteasome inhibitors. Proteasomes are large, non-lysosomal, multi-subunit protease complexes. They are characterised by evolutionarily conserved proteins and are present in Trypanosoma brucei [8], Trypanosoma cruzi [9], Toxoplasma gondii [10], Plasmodium spp. [11], Entamoeba histolytica and Entamoeba invadens [12], as well as in Leishmania mexicana [13]. A molecular similarity was found between the human 20S proteasome $\alpha$-type subunit and a Leishmania donovani cDNA clone [14] and between a gene encoding a 20S proteasome $\beta$ subunit in Plasmodium falciparum [15]. Proteasomes play a key role in differentiation and replication of protozoa, and lactacystin, a specific inhibitor of proteasomes, has a negative impact on the replication of L. mexicana promastigotes, although only at a high concentration [13].

We explored whether indinavir and saquinavir, two HIV protease inhibitors (PI) commonly used in infected individuals that share potent inhibitory effects on purified human proteasomes [6,7], have antileishmanial activity. 
The activity of DL- $\alpha$-difluoromethylornithine (DFMO), a polyamine analogue inhibiting protozoon ornithine decarboxylase [16], was also assessed.

\section{Materials and methods}

\subsection{Leishmaniae}

Leishmania major (LRC-L137 strain) and Leishmania infantum (MHOM/TN/80/IPT1 strain) were maintained in vitro at $25^{\circ} \mathrm{C}$ in Tobie's diphasic medium. Before use, promastigotes were grown in medium 199 (Invitrogen, CA, USA), modified as previously indicated [17], at $25^{\circ} \mathrm{C}$ for 4 days to reach late-log phase growth.

\subsection{In vitro studies}

The effects of the two PI, indinavir (Merck; a kind gift of S. Vella, Rome, Italy) and saquinavir (BS 00120083; a kind gift of Roche, Basel, Switzerland), on promastigotes of $L$. major and $L$. infantum were assessed by a method similar to that previously described [17]. Promastigotes were counted using a haemocytometer (Thoma chamber) and resuspended in fresh medium to a final concentration of $5 \times 10^{5}$ viable (showing motile behaviour and/or lack of staining after vital staining with trypan blue) promastigotes $/ \mathrm{mL}$. The two compounds were added to the cultures at final concentrations of $6.25,12.5,25$ and $50 \mu \mathrm{M}$ (starting from a $5 \mathrm{mM}$ solution in dimethylsulfoxide (DMSO) that was serially diluted in medium). Dilutions of DMSO corresponding to those used to prepare the drug solutions were assessed in parallel. DL- $\alpha$-DFMO (Sigma, Milan, Italy) was assayed at final concentrations of 3, 6, 12 and $24 \mathrm{mM}$. After 24, 48 and $72 \mathrm{~h}$ incubation at $25^{\circ} \mathrm{C}$ with occasional agitation, the number of viable, motile promastigotes was quantified. At the end of $24 \mathrm{~h}$ incubation, the reversibility of the effect was assessed by adding fresh complete medium at a 10:1 ratio; the same dilution was made for the control. After a further $48 \mathrm{~h}$ incubation, the number of live promastigotes was evaluated. The experiments were performed in triplicate and the standard deviations were $\leq 10 \%$ of the means obtained at each point. The $50 \%$ lethal dose $\left(\mathrm{LD}_{50}\right)$, i.e. the drug concentration that caused a 50\% reduction in survival/viability in comparison with that in identical cultures without the compound, was evaluated after $24 \mathrm{~h}$. This value was determined by non-linear regression analysis, by plotting the number of viable promastigotes versus log drug concentrations by use of GraphPad Prism 3 software.

\section{Results and discussion}

The results demonstrate that after 1 day of treatment, starting from a concentration of $6.25 \mu \mathrm{M}$, both indinavir and saquinavir induced $\sim 50 \%$ growth inhibition of L. major promastigotes (Table 1). At this time point, DMSO also exhibited a mild inhibitory activity, although only at dilutions corresponding to a drug concentration of $\geq 25 \mu \mathrm{M}$. After 2 days (and likewise after 3 days), the inhibitory effects of saquinavir on parasite growth persisted, ranging from $17 \%$ to $65 \%$ inhibition in a dose-dependent manner (Table 1). Similar results were obtained with indinavir, although the range of the inhibitory effect was lower. Conversely, at these time points the inhibition by DMSO virtually disappeared (Table 1). The antileishmanial activity was irreversible, and protozoa did not resume growth when subcultured in fresh medium. After incubation for $24 \mathrm{~h}$, the $\mathrm{LD}_{50}$ values of indinavir and saquinavir were $8.3 \pm 0.9 \mu \mathrm{M}$ and $7 \pm 0.7 \mu \mathrm{M}$, respectively. The inhibitory and lethal effects of the drugs on the $L$. infantum yield were less evident (Table 1), ranging from $5 \%$ to $34 \%$ in a dose-dependent manner. DMSO exhibited a limited inhibitory activity at higher concentrations $\left(\mathrm{LD}_{50}\right.$ values $>50 \mu \mathrm{M}$ for both protozoa). DL- $\alpha$-DFMO also had leishmanicidal effects, although only at high concentrations $\left(\mathrm{LD}_{50}\right.$ of $6 \mathrm{mM}$ for $L$. major and $15 \mathrm{mM}$ for $L$. infantum), higher than those values found on $L$. donovani [18].

Our findings demonstrate that indinavir and saquinavir have a dose-dependent leishmanicidal activity in vitro, which is greater on $L$. major than on L. infantum promastigotes. The inhibition of leishmanial growth produced by these com-

Table 1

Effect of indinavir and saquinavir on the in vitro growth of L. major and L. infantum promastigotes: values are reported as percentages of control grown in medium (considered 100\%)

\begin{tabular}{|c|c|c|c|c|c|c|c|c|c|c|c|c|c|}
\hline \multirow[t]{3}{*}{ Leishmania spp. } & \multirow[t]{3}{*}{ Days of treatment } & \multicolumn{12}{|c|}{ Dose } \\
\hline & & \multicolumn{3}{|c|}{$50 \mu \mathrm{M}$} & \multicolumn{3}{|c|}{$25 \mu \mathrm{M}$} & \multicolumn{3}{|c|}{$12.5 \mu \mathrm{M}$} & \multicolumn{3}{|c|}{$6.25 \mu \mathrm{M}$} \\
\hline & & I & $\mathrm{S}$ & $\mathrm{D}$ & I & $\mathrm{S}$ & $\mathrm{D}$ & I & $\mathrm{S}$ & $\mathrm{D}$ & I & $S$ & $\mathrm{D}$ \\
\hline \multirow[t]{3}{*}{ L. major } & 1 & $35^{\mathrm{a}}$ & $35^{\mathrm{a}}$ & 60 & $38^{\mathrm{a}}$ & $37^{\mathrm{a}}$ & 72 & $43^{a}$ & $41^{\mathrm{a}}$ & 100 & 53 & 51 & 100 \\
\hline & 2 & $47^{\mathrm{a}}$ & $35^{\mathrm{a}}$ & 71 & 66 & 60 & 88 & 80 & 71 & 100 & 93 & 80 & 100 \\
\hline & 3 & $48^{\mathrm{a}}$ & $45^{\mathrm{a}}$ & 83 & 78 & 58 & 95 & 85 & 77 & 100 & 100 & 83 & 100 \\
\hline \multirow[t]{3}{*}{ L. infantum } & 1 & 69 & 66 & 94 & 72 & 70 & 95 & 88 & 80 & 100 & 90 & 88 & 100 \\
\hline & 2 & 72 & 68 & 94 & 80 & 75 & 95 & 88 & 83 & 100 & 95 & 93 & 100 \\
\hline & 3 & 71 & 66 & 96 & 80 & 75 & 100 & 90 & 85 & 100 & 95 & 90 & 100 \\
\hline
\end{tabular}

I, indinavir; S, saquinavir; D, dimethylsulfoxide (DMSO) control at the same dilution used for drug solutions.

a Statistically significant difference, $P<0.05$. 
pounds may be due to their effects on proteasome activity. As observed on L. mexicana [13], these effects may depend on the stage of the parasite; for example, amastigotes developing within macrophages during human infection are more sensitive to lactacystin than promastigotes developing in culture medium. If the same applies to indinavir and saquinavir, their leishmanicidal effects should be higher in vivo than in vitro. Potent antiretroviral therapy with PI decreases the expression of virulence enzymes of Candida albicans [19]; furthermore, it has a negative impact on the yield of Cryptococcus neoformans [20], Pneumocystis carinii [21] and Cryptosporidium parvum [22]. In addition to the immune restoration, HIV-infected patients receiving PI may thus benefit from their direct inhibitory activity on a large array of microorganisms.

These results add new in vitro insights into the widespectrum efficacy of HIV PI and suggest studying their action on amastigote forms of leishmania within macrophages to validate their potential contribution against opportunistic infections in treated seropositive patients.

\section{References}

[1] Murray HW. Kala-azar as an AIDS-related opportunistic infection. AIDS Patient Care STDS 1999;13:459-65.

[2] Thakur CP, Narayan S, Ranjan A. Kala-azar (visceral leishmaniasis) and HIV coinfection in Bihar, India: is this combination increasing? J Acquir Immune Defic Syndr 2003;32:572-3.

[3] Morales MA, Cruz I, Rubio JM, et al. Relapses versus reinfections in patients coinfected with Leishmania infantum and human immunodeficiency virus type 1. J Infect Dis 2002;185:1533-7.

[4] Del Giudice P, Mary-Krause M, Pradier C, et al. Impact of highly active antiretroviral therapy on the incidence of visceral leishmaniasis in a French cohort of patients infected with human immunodeficiency virus. J Infect Dis 2002;186:1366-70.

[5] De La Rosa R, Pineda JA, Delgado J, et al. Incidence and risk factors for symptomatic visceral leishmaniasis among human immunodeficiency virus type 1 -infected patients from Spain in the era of highly active antiretroviral therapy. J Clin Microbiol 2002;40: $762-7$.

[6] Piccinini M, Rinaudo MT, Chiapello N, et al. The human 26S proteasome is a target of antiretroviral agents. AIDS 2002;16:693700.

[7] Piccinini M, Rinaudo MT, Anselmino A, et al. The HIV protease inhibitors nelfinavir and saquinavir, but not a variety of HIV reverse transcriptase inhibitors, adversely affect human proteasome function. Antivir Ther 2005;10:215-23.
[8] Li Z, Zou CB, Yao Y, et al. An easily dissociated 26S proteasome catalyzes an essential ubiquitin-mediated protein degradation pathway in Trypanosoma brucei. J Biol Chem 2002;277:15486-98.

[9] De Diego JL, Katz JM, Marshall P, et al. The ubiquitin-proteasome pathway plays an essential role in proteolysis during Trypanosoma cruzi remodeling. Biochemistry 2001;40:1053-62.

[10] Shaw MK, He CY, Roos DS, Tilney LG. Proteasome inhibitors block intracellular growth and replication of Toxoplasma gondii. Parasitology 2000;121:35-47.

[11] Gantt SM, Myung JM, Briones MR, et al. Proteasome inhibitors block development of Plasmodium spp. Antimicrob Agents Chemother 1998;42:2731-8.

[12] Makioka A, Kumagai M, Ohtomo H, Kobayashi S, Takeuchi T. Effect of proteasome inhibitors on the growth, encystation, and excystation of Entamoeba histolytica and Entamoeba invadens. Parasitol Res 2002;88:454-9.

[13] Robertson CD. The Leishmania mexicana proteasome. Mol Biochem Parasitol 1999;103:49-60.

[14] Christensen CB, Jorgensen L, Jensen AT, et al. Molecular characterization of a Leishmania donovani cDNA clone with similarity to human 20S proteasome $\alpha$-type subunit. Biochim Biophys Acta 2000;1500:77-87.

[15] Li GD, Li JL, Mugthin M, Ward SA. Molecular cloning of a gene encoding a $20 \mathrm{~S}$ proteasome beta subunit from Plasmodium falciparum. Int J Parasitol 2000;30:729-33.

[16] Calonge M, Cubria JC, Balana-Fouce R, Ordonez D. Putrescine active uptake system in the trypanosomatid Crithidia fasciculata. Biol Chem Hoppe Seyler 1996;377:233-8.

[17] Savoia D, Avanzini C, Allice T, Callone E, Guella G, Dini F. Antimicrobial activity of euplotin $\mathrm{C}$, the sesquiterpene taxonomic marker from the marine ciliate Euplotes crassus. Antimicrob Agents Chemother 2004;48:3828-33.

[18] Mukhopadhyay R, Madhubala R. Effects of bis(benzyl)polyamine analogs on Leishmania donovani promastigotes. Exp Parasitol 1995;81:39-46.

[19] De Bernardis F, Tacconelli E, Mondello F, et al. Anti-retroviral therapy with protease inhibitors decreases virulence enzyme expression in vivo by Candida albicans without selection of avirulent fungus strains or decreasing their anti-mycotic susceptibility. FEMS Immunol Med Microbiol 2004;41:27-34

[20] Blasi E, Colombari B, Orsi CF, et al. The human immunodeficiency virus (HIV) protease inhibitor indinavir affects the opportunistic fungal pathogen Cryptococcus neoformans. FEMS Immunol Med Microbiol 2004;42:187-95.

[21] Atzori C, Clerici M, Trabattoni D, et al. Assessment of immune reconstitution to Pneumocystis carinii in HIV-1 patients under different highly active antiretroviral therapy regimens. J Antimicrob Chemother 2003;52:276-81.

[22] Hommer V, Eichholz J, Petry F. Effect of antiretroviral protease inhibitors alone, and in combination with paromomycin, on the excystation, invasion and in vitro development of Cryptosporidium parvum. J Antimicrob Chemother 2003;52:359-64. 